\title{
Diabetic macular edema grading in retinal images using vector quantization and semi-supervised learning
}

\author{
Fulong Ren ${ }^{\mathrm{a}, \mathrm{b}}$, Peng $\mathrm{CaO}^{\mathrm{a}, \mathrm{b}}$, Dazhe Zhao ${ }^{\mathrm{a}, \mathrm{b}}$ and Chao Wan ${ }^{\mathrm{c}, *}$ \\ ${ }^{a}$ School of Computer Science and Engineering, Northeastern University, Shenyang, Liaoning, China \\ ${ }^{\mathrm{b}}$ Key Laboratory of Medical Image Computing of Ministry of Education, Northeastern University, \\ Shenyang, Liaoning, China \\ ${ }^{\mathrm{c}}$ Department of Ophthalmology, The First Hospital of China Medical University, Shenyang, Liaoning, \\ China
}

\begin{abstract}
.
BACKGROUND: Diabetic macular edema (DME) is one of the severe complication of diabetic retinopathy causing severe vision loss and leads to blindness in severe cases if left untreated.

OBJECTIVE: To grade the severity of DME in retinal images.

METHODS: Firstly, the macular is localized using its anatomical features and the information of the macula location with respect to the optic disc. Secondly, a novel method for the exudates detection is proposed. The possible exudate regions are segmented using vector quantization technique and formulated using a set of feature vectors. A semi-supervised learning with graph based classifier is employed to identify the true exudates. Thirdly, the disease severity is graded into different stages based on the location of exudates and the macula coordinates.

RESULTS: The results are obtained with the mean value of 0.975 and 0.942 for accuracy and F1-scrore, respectively.

CONCLUSION: The present work contributes to macula localization, exudate candidate identification with vector quantization and exudate candidate classification with semi-supervised learning. The proposed method and the state-of-the-art approaches are compared in terms of performance, and experimental results show the proposed system overcomes the challenge of the DME grading and demonstrate a promising effectiveness.
\end{abstract}

Keywords: Retinal images, diabetic macular edema, exudate detection, classification

\section{Introduction}

Diabetic macular edema (DME), an advanced symptom of Diabetic Retinopathy (DR), is the major causes of vision loss in diabetic patients and even leads to blindness in severe cases [1]. It is caused by one of the DR lesions, called as exudate, encroaching on the macula in the retina, which is the part of the eye that is responsible for the clear, sharp and detailed vision [2]. The exudates appear as yellow or white structures with varying size, shape and position and often occur in clusters, and occur when the fluid rich in fat begins to leak from the damaged blood vessels and gets deposited. When any exudate occurs near

\footnotetext{
${ }^{*}$ Corresponding author: Chao Wan, Department of Ophthalmology, The First Hospital of China Medical University, Shenyang, Liaoning, China. E-mail: renfl@ neusoft.com.

0928-7329/18/\$35.00 (c) 2018 - IOS Press and the authors. All rights reserved

This article is published online with Open Access and distributed under the terms of the Creative Commons Attribution NonCommercial License (CC BY-NC 4.0).
} 
Table 1

Criterion for grading of diabetic macular edema

\begin{tabular}{lll}
\hline Grade & Grading criterion & Class \\
\hline 0 & No visible exudates & Normal \\
1 & Shortest distance between macula and exudates $>$ one optic disc diameter & Non-CSME \\
2 & Shortest distance between macula and exudates $\leqslant$ one optic disc diameter & CSME \\
\hline
\end{tabular}

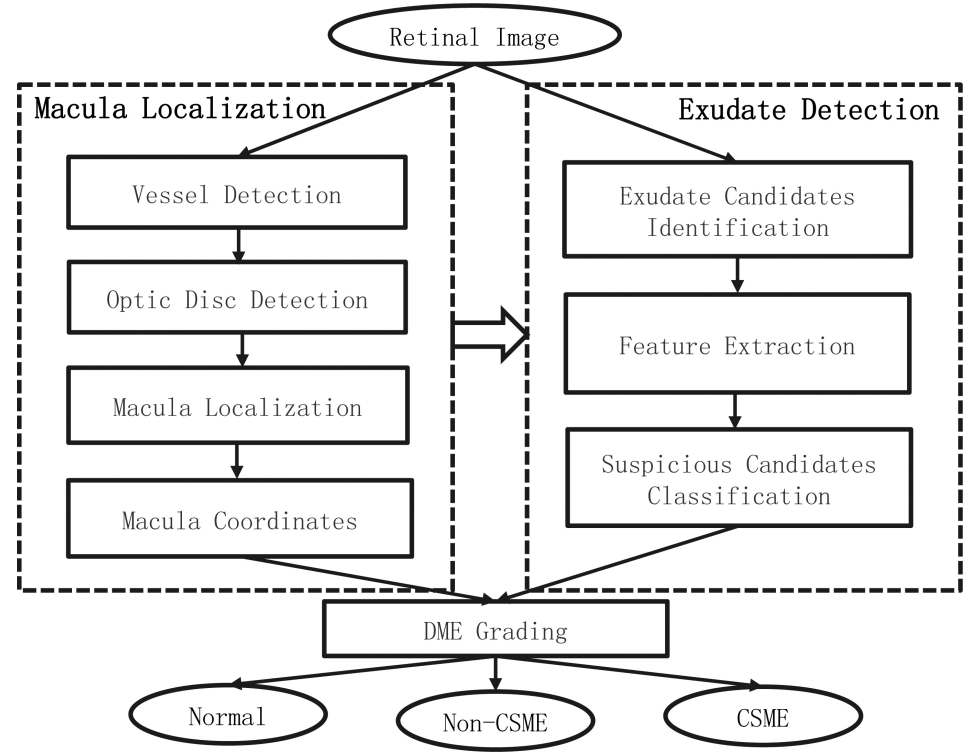

Fig. 1. The flowchart of the proposed algorithm for grading DME.

or on the macula, the macula begins to thicken, swell and the patient's vision becomes to blur. In the retinal images, these DME often has no early warning signs and a patient doesn't realize his condition until the disease is intensified, so early diagnosis and treatment are critical to prevent progress of disease and vision loss. Currently, the assessment of the severity of DME is based on the distances of the exudate to the macula. The closer the exudate is to the macular, the more the risk increases. DME consists of two types: non-clinically significant macular edema (non-CSME) and clinically significant macular edema (CSME) [2]. The definition of grading criteria is shown in Table 1 in the MESSIDOR database (http://messidor.crihan.fr/index-en.php, updated 2016 November 8). DME are normally detected and graded manually from fundus photographs by clinicians with a time-consuming and laborious intensive process. In addition, the increased prevalence of diabetes and the scarcity of eye care specialists cause a bottleneck to meet the requirement of DME screening. An automatic screening system for grading of the severity of DME is a potential solution to this problem.

In a computer aided diagnostic system for DME, automated detection of macula and exudates is a vital task [2-4]. Recently, many kernel based methods with faster optimization speed or stronger generalization performance have been proposed and investigated with theoretic analysis and experimental evaluation [5,6]. For example, SVM is used to grade the diabetic maculopathy in [2]. An improved fuzzy C-means along with SVM are utilized for the detection of exudates [7]. Moreover, neural network-based approaches are proposed to automatically detect exudates in retina images [8]. Although a number of methods have been proposed for the macula and exudate detection, there are still some unsolved issues. The major issue is the existence of false positive regions for exudates and macula. Most of the exu- 


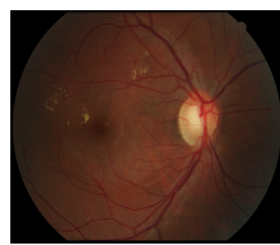

(a)

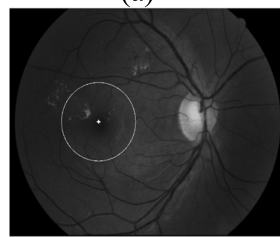

(e)

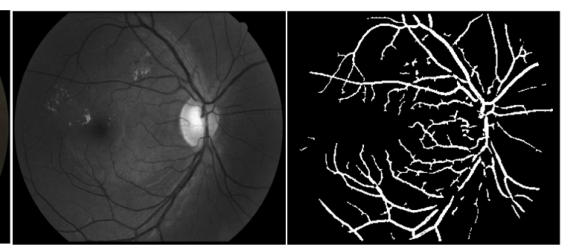

(b)

(c)

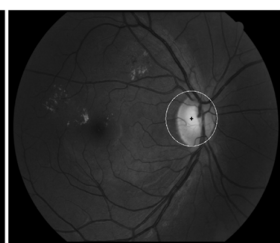

(d)

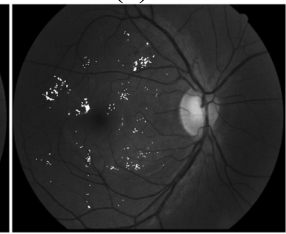

(f)

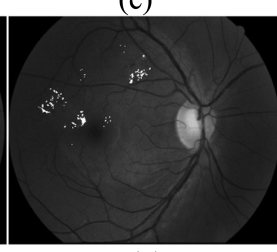

(g)

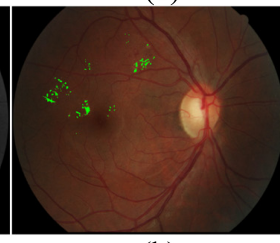

(h)

Fig. 2. An example of the proposed procedure. (a) Original image, (b) the green channel image, (c) outputs of main vessel segmentation, (d) OD localization and defection, (e) macula localization and coordinates, (f) outputs of exudate candidates, (g) outputs of exudate classification, (h) the final outputs of exudate in RGB image.

date detection methods in DME grading system is generally based on supervised learning algorithms, which need sufficient training dataset with label information. However, there is no publicly available dataset with label of both grading and exudate lesions. For example, the MESSIDOR dataset contains the grading information for DME but no ground-truth location of exudate, whereas E-ophtha dataset (http://www.adcis.net/en/Download-Third-Party/E-Ophtha.html, updated 2014 May 25) contains the location information of exudate without the grading label. In order to deal with the issue of such weakly labeled data, an automatic screening system with semi-supervised learning [9] for DME grading is presented in this study. In the proposed system, multiple features of macula are considered to improve the accuracy of macula detection. Then, during the exudate detection phase a novel vector quantization based detection algorithm is proposed. Finally, a semi-supervised learning method is used to leverage the auxiliary labeled data from a source dataset to improve the generalization ability of classification.

\section{Proposed methodology}

\subsection{Overview}

The proposed screening system for grading of DME presented can be schematically described by the block diagram in Fig. 1. It consists of three main processing stages: 1) macula localization, containing several main retina tissue detection, i.e. vessel, optic disc and macula; 2) exudate detection, containing three steps of exudate candidates' identification, feature extraction and suspicious candidates classification; 3) grading of diabetic macular edema, according to macular coordinates and location of exudates to classify the input image into one of the status: normal, Non-CSME and CSME. Once the exudates and macular coordinates are obtained, the grading of DME can be done according to the grading criteria shown in Table 1. The following sections mainly describe the detection methods of macula and exudate in detail.

\subsection{Macula localization}

In the retinal image, macula is the darkest circular region and locates at a specific distance from optic disc (OD). According to the criterion, many methods are proposed [10]. However they fail to detect the 


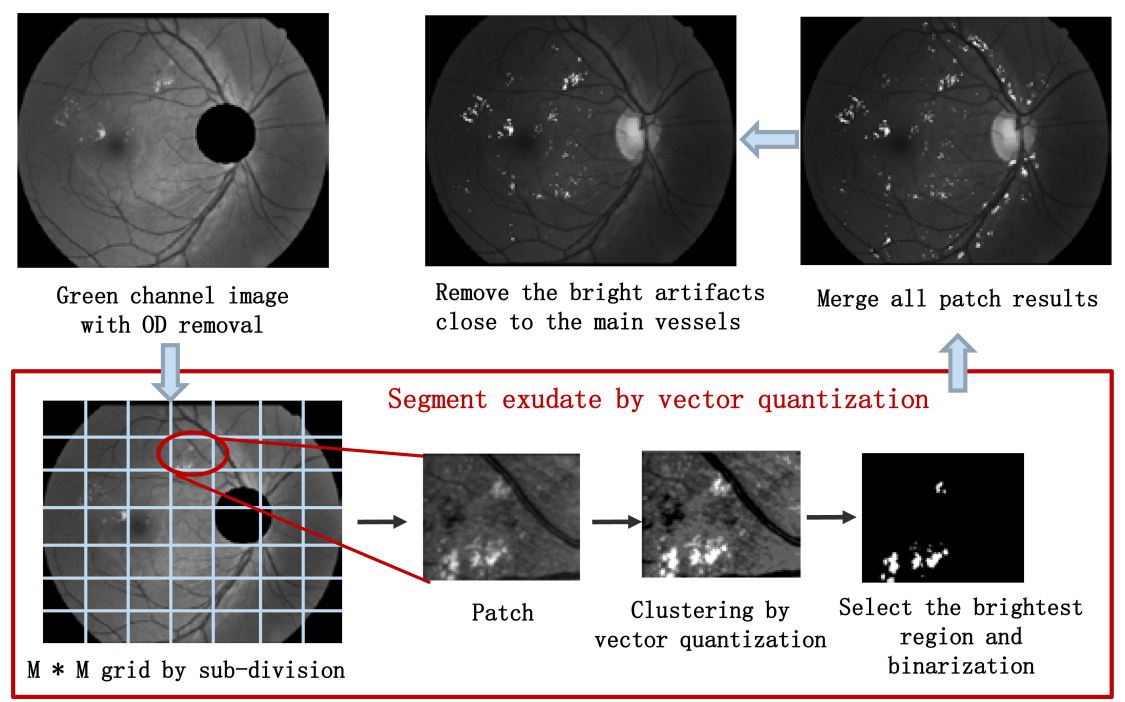

Fig. 3. The procedure of the exudate candidates' identification.

macula when the retina contains large dark lesions, i.e. hemorrhages. In this study, a method based on considering multiple features is proposed and achieves a better performance. It is critical to detect the retinal blood vessels and the optic disc prior to localizing the macula.

The method [11] was adopted to assess the size of the OD, and a morphological top-hat operation combined with thresholding technique is employed to segment the main vessels of the retina in the green channel. Then, the OD is detected by using a line operator filter. Following this, a candidate region of interest is defined as the ring area with a radius of 2 to 3 times as the optic disc diameter (DD) from the center of the optic disc [10]. Within the ring area, a circle template with a diameter of about $2 / 3$ of DD [12] is designed and applied to select macula candidates by template matching. Furthermore, two statistic values in the $R O I$, the mean intensity $\left(M_{R O I}\right)$ and the standard deviation of the intensities $\left(\sigma_{R O I}\right)$, are introduced for macula candidate identification. The products of $M_{R O I}$ and $\sigma_{R O I}$ are sorted in ascending order and the ROIs with the top three values are identified as macula candidates. The true macula is selected according to the fact that no blood vessels locate inside the macula. The results of the aforementioned procedure are illustrated in Fig. 2a-e.

\subsection{Vector quantization for exudate candidates' identification}

In retinal images, which are usually two-dimensional 24-bit color images obtained through a digital fundus camera, exudates generally appear as bright regions with variable brightness, sizes, locations and shapes. Accurate exudate segmentation is a challenging task due to the large variety in size, intensity, shape and contrast. In this study, the vector quantization (VQ) technique is introduced to segment the exudates. VQ [13] is an efficient technique for data compression, and it can be defined as a mapping function that maps $k$-dimensional vector space into a finite set $C B=\left\{C_{1}, C_{2}, C_{3}, \ldots, C_{N}\right\}$. The set $C B$ is called a codebook consisting of $N$ code vectors and each code vector $C_{i}=\left\{c_{i 1}, c_{i 2}, c_{i 3}, \ldots, c_{i k}\right\}$ is $k$-dimensional. It works by dividing a large set of points into groups having approximately the same number of points closest to them if these points are large enough to ensure the probability distribution of points. The groups are normally generated by clustering algorithms, and each group is represented by its centroid as in K-means. In our method, the Linde-Buzo-Gray (LBG) algorithm is used to generate 
the codebook. More specifically, an initial vector $C^{0}$ is set as the average of the entire training vectors. By adding a small constant error $\epsilon>0$, the vector is then split into two vectors $C_{1}^{0}=(1+\epsilon) C^{0}$ and $C_{2}^{0}=(1-\epsilon) C^{0}$. The iterative algorithm is run with the two vectors as the initial codebook, and the process is repeated until the desired size of the codebook is obtained.

Intensity inhomogeneity and uneven illumination hinder the segmentation of the exudates. To solve it, a local region-based segmentation is adopted to segment the image into a number of homogeneous sub-images before the clustering. A region-based segmentation scheme is presented in Fig. 3. Firstly, the OD is removed from the green channel image. Then, the image is partitioned into patches with same size by the grid of $M * M$. Subsequently, the VQ clustering is carried out on each patch to obtain the codebook and the image is reconstructed using the codebook. The grid of $M * M$ for dividing the image into non overlapping patches is empirically set to $7 * 7$, and the size of codebook is also empirically set to 8 . Merging the results of all the patches is done followed by selecting brightest region and binarization on each patch. The segmentation process by VQ is defined as Eq. (1).

$$
I_{\text {seg }}=\sum_{i=0}^{n} V\left(P_{i}\right)
$$

where $I_{\text {seg }}$ denotes the exudate result of the whole image by VQ, $V(\cdot)$ represents the VQ segmenting to the exudate for the input patch, $P_{i}$ is the $i$ patch of the all non-overlapping patches and $n$ is the number of all patch. Finally, the bright ROI close to the main vessels are removed by the method in [1], since some bright structures such as the reflections and nerve fibers within the vessels have similar appearance to exudates and can lead to high false positive rate. The final result of exudate candidates is illustrated in Fig. 2f.

\subsection{Semi-supervised learning for exudate candidates classification}

Features extraction is critical for classification. Since exudates appear as yellow or white areas with different size and shape in the retina, color features are extracted from gray level image, the $G$ as well as R channels of RGB color space and the L channel of CIElab color space. Exudates have strong and sharp edges, thus the mean, standard deviation, minimum, maximum of the gradient are calculated on the candidate regions to capture the local gradient information. Entropy is also calculated by including all pixels in bounding square around candidate region to measure the context information of candidate regions. In addition, another two contextual features, the distance to the closest candidate and the number of the nearby candidates, based on the spatial relation with surrounding similar lesions are also taken into account since exudates often occur in clusters and a particular slightly suspect candidate has a higher chance of being a true exudate when another more obvious exudate is present nearby. In total, a 23 dimensional feature is extracted for each candidate exudate.

After extracting features for all candidates, the following phase is to classify them into exudates or non-exudates by machine learning. The traditional classification methods require a sufficiently large number of training datasets with label information. Nevertheless, manually annotating labels for these exudate candidates is difficult. There is no such a publicly available database containing both ground truth of DME diagnosis at an image label and exudate segmentation at a lesion label. Although training a supervised classifier can be done on another datasets with ground truth of exudate, the difference between datasets may lead to a decline in the performance of DME grading. Fortunately, semi-supervised learning (SSL) can provide a suitable solution for this problem. SSL aims to utilize unlabeled data and the limited available labeled data to learn better supervised models with good generalization ability. This study adopt 
Table 2

The comparison among different $l p$ using the proposed method for the DME grading on MESSIDOR dataset

\begin{tabular}{ccccc}
\hline lp & Accuracy & Sensitivity & Specificity & F1-score \\
\hline $20 \%$ & $0.889 \pm 0.009$ & $0.797 \pm 0.011$ & $0.915 \pm 0.016$ & $0.786 \pm 0.009$ \\
$40 \%$ & $0.912 \pm 0.010$ & $0.842 \pm 0.013$ & $0.931 \pm 0.021$ & $0.831 \pm 0.011$ \\
$60 \%$ & $0.945 \pm 0.015$ & $0.894 \pm 0.012$ & $0.958 \pm 0.019$ & $0.887 \pm 0.013$ \\
$80 \%$ & $0.975 \pm 0.010$ & $0.946 \pm 0.011$ & $0.982 \pm 0.017$ & $0.942 \pm 0.009$ \\
$100 \%$ & $0.927 \pm 0.012$ & $0.865 \pm 0.010$ & $0.943 \pm 0.020$ & $0.855 \pm 0.010$ \\
\hline
\end{tabular}

a graph-based semi-supervised learning approach in [9] which defines a similarity graph where vertices are the labeled as well as unlabeled examples, and edges indicate the similarities of pairs of vertices. Let $V$ be a set of vertices with $n=|V|$, and the edge weights matrix is defined by $W \in R_{n} \times R_{n}$. Given two vertices $x_{i}$ and $x_{j}$, the edge weight $W_{i, j}$ indicates the similarity of these instances and is calculated using Gaussian RBF kernel: $W_{i, j}=\exp \left(\gamma|| x_{i}-x_{j} \|^{2}\right)$, where $\gamma$ is a kernel parameter. With the help of the graph structure, the unlabeled vertices can be accurately classified by using a small amount of labelled examples. Given a labelled instances $\left(x_{i}, y_{i}\right)$ and a unlabeled instances $x_{j}$, where $i \in(1, \ldots, L)$ and $j \in(L+1, \ldots, L+U)$, and a graph $\boldsymbol{G}$ is denoted as a $(L+U) \times(L+U)$ correlation matrix. The graph-based semi-supervised learning method assumes that a function $f$ can be found so that it is close to the given labels on the labeled examples and it is also smooth on the whole graph. This framework is based on semi-supervised learning with graph embeddings, and a feed-forward neural networks is used to represent embeddings of instances. Given the input feature vector $x$, the output function of the $k$-th hidden layer of the network is defined as $h^{k}(x)=\operatorname{ReLU}\left(a^{k} h^{k-1}(x)+b^{k}\right)$, where $a^{k}$ and $b^{k}$ are parameters of the $k$-th layer, and $h^{0}(x)=x, \operatorname{ReLU}(x)=\max (0, x)$. Given a graph $\boldsymbol{G}$, the objective function $f$ of this framework can be written as:

$$
f=f_{S L}+\lambda f_{U L}
$$

where $f_{S L}$ is a supervised loss of predicting the labels, $f_{U L}$ is an unsupervised loss of predicting the graph context, and $\lambda$ is a regularization parameter.

The first term is the log conditional probability for labeled data, which is defined as:

$$
f_{S L}=-\frac{1}{L} \sum_{i=1}^{L} \log p\left(y_{i} \mid x_{i}\right), \text { and } p(y \mid x)=\frac{\exp \left[h^{k}(x)^{T}, h^{l}(x)^{T}\right] w_{y}}{\sum_{y^{\prime}} \exp \left[h^{k}(x)^{T}, h^{l}(x)^{T}\right] w_{y^{\prime}}}
$$

where $w$ represents the weighting parameter for the class label $y$. The representation $h^{k}(x)$ from the $k$ layers on the input feature vector $x$ and the representation $h^{l}(x)$ from the $l$ layers on the embedding are concatenated, and fed into a softmax layer to predict the class labels of the instances.

The unsupervised loss is usually defined with the $\log \operatorname{loss}-\log p(c \mid i)$. Given an instance $i$ and its context $c$, the objective of the unsupervised loss is formulated as minimizing the log loss of predicting the context $c$ using the embedding of an instance as input features. It uses the embedding of a vertex to predict the context in the graph, where the context is generated by random walk. The distribution $p(i c \delta)$ is defined for a sampling precess and is conditioned on labels $y_{1: L}$ and the graph $\boldsymbol{G}$. A sampling $(i c \delta)$ is done from the distribution. Given $(i c \delta)$, the cross entropy loss of classifying the pair $(i c)$ to a binary label $\delta$ can be minimized as $\Pi_{(i, c, \delta)} \log \mu\left(\delta a_{C}^{T} e_{i}\right)$, where $\Pi(i c \delta)$ is an indicator function that outputs 1 when the argument is true, otherwise $0, \mu(\cdot)$ is the sigmoid function, $a^{\prime} s$ represent the weighting parameters, and $e_{i}$ is the embedding of instance $i$. The embedding $e$ is used with $l_{1}$ layers of the network, and the second term in Eq. (2) can be written as

$$
f_{U L}=\Pi_{(i, c, \delta)} \log \mu\left(\delta a_{C}^{T} h^{l_{1}}\left(x_{i}\right)\right)
$$


Table 3

The comparison among the state-of-the-art semi-supervised classifiers and DME grading methods on MESSIDOR dataset

\begin{tabular}{lcccc}
\hline Method & Accuracy & Sensitivity & Specificity & F1-score \\
\hline Neural network & $0.909 \pm 0.010$ & $0.830 \pm 0.015$ & $0.931 \pm 0.011$ & $0.820 \pm 0.012$ \\
Self-training [14] & $0.951 \pm 0.015$ & $0.894 \pm 0.011$ & $0.961 \pm 0.014$ & $0.891 \pm 0.018$ \\
Co-training [15] & $0.960 \pm 0.009$ & $0.890 \pm 0.012$ & $0.960 \pm 0.009$ & $0.879 \pm 0.013$ \\
Lim et al. [3] & 0.852 & 0.809 & 0.902 & Not reported \\
Sreejini and Govindan [4] & 0.945 & 0.91 & 0.98 & Not reported \\
Akram et al. [2] & 0.973 & 0.926 & 0.978 & Not reported \\
Proposed & $0.975 \pm 0.010$ & $0.946 \pm 0.011$ & $0.982 \pm 0.017$ & $0.942 \pm 0.009$ \\
\hline
\end{tabular}

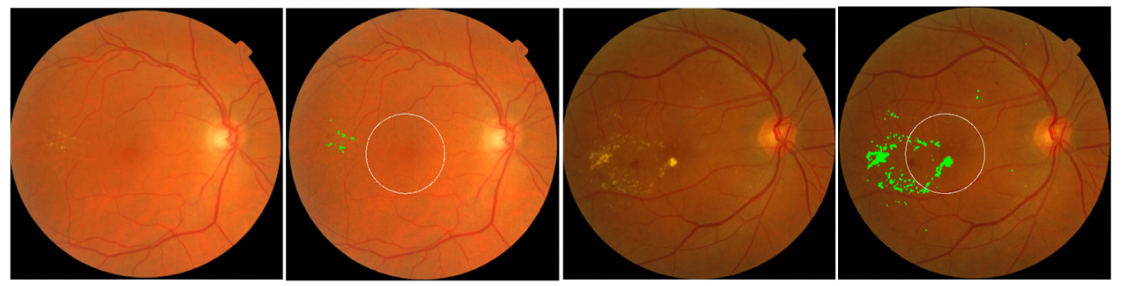

(a)

(b)

(d)

Fig. 4. Examples of macular edema detection for MESSIDOR database. (a) and (b): Original and result of exudate detection and macular coordinates images of a non-CSME example, (c) and (d): Original and result of exudate detection and macular coordinates images of a CSME example, the detected exudates are labelled in green.

Therefore, according to Eqs (3) and (4), the function $f$ in inductive learning can be written as follow.

$$
f=-\frac{1}{L} \sum_{i=1}^{L} \log p\left(y_{i} \mid x_{i}\right)-\lambda \Pi_{(i, c, \delta)} \log \mu\left(\delta a_{C}^{T} h^{l_{1}}\left(x_{i}\right)\right)
$$

In the optimization phase, stochastic gradient descent (SGD) is applied to train the above model and the result images of suspicious exudates classification are shown as Fig. $2 \mathrm{~g}$ and $\mathrm{h}$.

\section{Results and discussion}

The performance of grading the severity of DME is evaluated on 500 images from the publicly available MESSIDOR dataset which only provides the DME diagnosis label. These images were captured at different sizes: $1440 * 960,2240 * 1488$ or $2304 * 1536$ pixels and were 8 bits per color plane. Moreover, another public available database, E-ophtha EX dataset which is used as labeled dataset $(L)$ with its provided ground truth for exudate segmentation, is combined with MESSIDOR dataset which is used as unlabeled dataset $(U)$ to train the SSL classifier. E-ophtha EX contains 47 images with exudate regions and 35 exudate-free images. Figure 4 illustrates the result of the exudate detection on non-CSME image and CSME images at lesion level. A region is considered as true positive exudate if $50 \%$ of its pixels overlap with the region given in ground truth. It is observed that small and faint exudates is also well segmented by employing the proposed method.

The performance of the proposed method is assessed with respect to sensitivity, specificity, accuracy and F1-score (balanced F Score).

$$
\begin{aligned}
& \text { Sensitivity }=T P /(T P+F N), \text { Specificity }=T N /(T N+F P) \\
& \text { Accuracy }=(T P+T N) /(T P+F P+T N+F N)
\end{aligned}
$$




$$
\text { F1-score }=2 * P * R /(P+R), P=T P /(T P+F P), R=T P /(T P+F N)
$$

where $T P$ is true positives, $F P$ is false positives, $F N$ is false negatives, $T N$ is true negatives, $P$ is precision and $R$ is recall.

Ten trials of 5-fold nested cross-validation are adapted to evaluate the performance of proposed algorithm. The parameters ( $\lambda$ and the number of hidden layers) are empirically chosen for the best performance. In each trail, $20 \%$ of $U$ is used as testing set, and the remaining $80 \%$ of $U$ is used as the training set. For the labeled training set $L$, it is partitioned into two parts according to a given percentage of labeled data $(l p)$. Suppose $l p$ is $10 \%$, then $10 \%$ of the $L$ data is randomly sampled and combined with $80 \%$ of $U$ to construct the training set. Different values were set to $l p$ in the experiments ranging from $20 \%$ to $100 \%$. Table 2 shows the results with respect to different value of $l p$. The best value of $l p$ is $80 \%$ from the result.

In Table 3, the performances of DME grading are shown by using supervised neural network classifier, self-training, co-training, the proposed classifier and the methods of the existing system of DME grading. In all the SSL methods neural network is chosen as the base classifier. For three comparable SSL methods, $l p$ is set to $80 \%$. From the results in Table 3, the proposed method provides clearly the best performance.

\section{Conclusion}

Diabetic macular edema (DME) is an advanced symptom of diabetic retinopathy and can lead to irreversible vision loss. In this study, a computerized assist system for automated macular edema grading is presented. The main contributions of proposed system are macula localization, exudate candidate identification with vector quantization and exudate candidate classification with semi-supervised learning. The proposed method and the state-of-the-art approaches are compared in terms of performance, and experimental results show our system overcomes the challenge of the DME grading and demonstrate a promising effectiveness.

\section{Acknowledgments}

This research was supported by the National Natural Science Foundation of China (No. 61502091), the Fundamental Research Funds for Shenyang Municipal Science and Technology Bureau (No. 17134-8-00) and the Fundamental Research Funds for the Central Universities (No. N161604001 and N150408001).

\section{Conflict of interest}

None to report.

\section{References}

[1] Zhang X, Thibault G, Decencière E, et al. Exudate detection in color retinal images for mass screening of diabetic retinopathy. Medical Image Analysis 2014; 18(7): 1026. 
[2] Akram MU, Akhtar M, Javed MY. An automated system for the grading of diabetic maculopathy in fundus images. International Conference on Neural Information Processing, Springer-Verlag 2012; 36-43.

[3] Lim ST, Zaki WMDW, Hussain A, et al. Automatic classification of diabetic macular edema in digital fundus images. Humanities, Science and Engineering, IEEE 2012; 265-269.

[4] Sreejini KS, Govindan VK. Severity grading of DME from retina images: A combination of PSO and FCM with Bayes classifier. International Journal of Computer Applications 2014; 81(16): 11-17.

[5] Gu B, Sheng VS. A robust regularization path algorithm for $\nu$-support vector classification. IEEE Transactions on Neural Networks and Learning Systems 2016.

[6] Gu B, Sheng VS, Tay KY, et al. Incremental support vector learning for ordinal regression. IEEE Transactions on Neural Networks and Learning Systems 2015; 26(7): 1403-1416.

[7] Zhang X, Chutatape O. Top down and bottom up strategies in lesion detection of background diabetic retinopathy. IEEE Computer Society Conference on Computer Vision and Pattern Recognition 2005; 422-428.

[8] Schaefer G, Leung E. Neural networks for exudate detection in retinal images. International Conference on Advances in Visual Computing 2007; 298-306.

[9] Yang Z, Cohen WW, Salakhutdinov R. Revisiting semi-supervised learning with graph embeddings. ICML 2016; 40-48.

[10] Sinthanayothin C, Boyce JF, Cook HL, et al. Automated localisation of the optic disc, fovea, and retinal blood vessels from digital colour fundus images. British Journal of Ophthalmology 1999; 83(8): 902-910.

[11] Ren F, Li W, Yang J, et al. Automatic optic disc localization and segmentation in retinal images by a line operator and level sets. Technology \& Health Care Official Journal of the European Society for Engineering \& Medicine 2016; 24(s2).

[12] Grading diabetic retinopathy from stereoscopic color fundus photographs - an extension of the modified Airlie House classification. ETDRS report number 10, Early Treatment Diabetic Retinopathy Study Research Group, Ophthalmology 1991; 98: 786.

[13] Jaspreet K, Amita C. Comparison of several contrast stretching techniques on acute Leukemia images. International Journal of Engineering and Innovative Technology (IJEIT) 2012; 2(1): 332-335.

[14] Yarowsky D. Unsupervised word sense disambiguation rivaling supervised methods. Proceedings of Annual Meeting of the Association for Computational Linguistics 1995; 189-196.

[15] Blum A, Mitchell T. Combining labeled and unlabeled data with co-training. Eleventh Conference on Computational Learning Theory, ACM 1998; 92-100. 